\title{
Remote Sensing-Based Analysis of Landscape Pattern Evolution in Industrial Rural Areas: A Case of Southern Jiangsu, China
}

\author{
Yifan Zhu ${ }^{1}$, Chengkang Wang ${ }^{1, *(1)}$ and Takeru Sakai ${ }^{2}$ \\ 1 College of Landscape Architecture, Nanjing Forestry University, Nanjing 210037, China; \\ zhuyifan1997@outlook.com \\ 2 Campus Planning Office, Kyushu University, Fukuoka 819-0383, Japan; sakai@kyudai.jp \\ * Correspondence: chengkang.wang@njfu.edu.cn; Tel.: +86-18951841571
}

Received: 9 August 2019; Accepted: 10 September 2019; Published: 12 September 2019

check for updates

\begin{abstract}
With the rapid economic development of industrial rural areas in Southern Jiangsu, the rural landscape and ecological environment of these industrial rural areas are getting damaged. Based on GIS and RS techniques, Landsat Satellite remote sensing images from 1981, 1991, 2001, 2011 and 2018 were collected for Jiangyin, Zhangjiagang, Changshu and Kunshan, to extract landscape pattern indexes and spatial distribution data. Landscape pattern indexes of the patch-class level and landscape level from each year were calculated by FRAGSTATS. After analyzing and comparing landscape pattern variation of five years, progress, characteristics and driving forces of landscape pattern evolution were explored. At the patch-class level, construction land had continuously encroached on green and cultivated land, exhibiting trends of expansion and centralization. At the landscape level, the number of small patches and degree of landscape fragmentation generally increased. The direct cause of landscape pattern evolution in industrial rural areas of Southern Jiangsu was the encroachment and segmentation of green and cultivated land by construction land, and the dominant factors driving the changes in construction land in the industrial rural areas of Southern Jiangsu were the effects of land and population aggregation exerted by the development of township enterprises and rural industries.
\end{abstract}

Keywords: landscape pattern; industrial rural area; rural landscape; landscape ecology; southern Jiangsu

\section{Introduction}

Industrial rural areas are clusters of rural land with high industrial development, a large number of industrial areas and industrial enterprises based on raw material collection and processing-manufacturing industries, where the industrial output value accounts for the highest proportion of the total rural community assets [1]. During the early period of economic reform in China during the 1980s, the location of the rural areas in Southern Jiangsu Province facilitated the rapid development of township enterprises based on manufacture, which led to the establishment of a unique industry-dominated rural economic development pathway referred to as the "Southern Jiangsu Model" and the formation of large industrial rural areas. The Southern Jiangsu Model is one of the most representative models of the urbanization process in China and has certain research value. However, as development in Southern Jiangsu occurred during the early period of urbanization, there was a lack of preceding examples that could provide a reference and theoretical guidance for sustainable development. Consequently, the original, natural appearance of these rural landscapes was destroyed, diminishing their unique characteristics and rurality [2]. Additionally, the rapid 
urbanization of peripheral areas also caused the continuous extension of urban landscapes into rural areas [3]. Under the joint influence of the aforementioned internal and external factors, the landscape patterns of the industrial rural areas in Southern Jiangsu gradually evolved to their present state, which is characterized by the widespread loss of traditional rural landscapes. Additionally, the encroachment of urban construction on rural land and the commingling of residential and industrial zones in rural construction areas have caused the continuous deterioration of the environments surrounding human settlements in these areas [4]. With the proposal of the ecological civilization construction concept and rural revitalization strategy, strategies to mitigate the destruction of rural landscapes and assimilation effects of urban-rural integration, enhance the environment surrounding rural settlements and promote the sustainable development of rural areas have become the focus of research on Chinese rural areas [5].

Industrial rural development has resulted in the evolution of rural functions and the modification of industrial rural structures on a global scale. Although industrial rural and manufacturing models had previously promoted economic development and increased the income of rural residents, they significantly impacted land use patterns and rural environments, which is unfavorable for sustainable development [6]. Recent major economic and social reforms have driven the development of tourism, which has, in turn, promoted the development of the national economy through industrial, agricultural, construction, transportation and trade activities $[7,8]$. The development of the tourism sector has profoundly influenced the transformation of various economic, social and cultural factors, leading to the establishment of the rural tourism market and the consolidation of the tourism sector while also promoting sustainable development and environmental conservation $[9,10]$. Rural tourism aims to sustain rural architecture and landscapes, thereby promoting creative activities related to the natural and historical backgrounds of rural areas for the appropriate development of these rural landscapes and their cultural heritage. This is also a major pathway in the sustainable development of rural areas [11,12].

After sorting out the relevant research results, it is found that researchers have studied the spatial and temporal distribution characteristics of carbon emissions [13], industrial pollution [14], ecological sensitivity [15] and the natural landscape level [16] in the industrial rural areas in Southern Jiangsu by using the methods of composite analysis, buffer analysis and data weighted overlay analysis in the GIS platform. These studies have achieved effective results, but generally there are some shortcomings in data and methods, such as small research scope, low accuracy of data and a lack of macro-control of the development of urban and rural areas for the region as a whole.

Previous studies indicated that landscape pattern indexes calculated based on land-use categories directly reflect the characteristics of changes to a certain region from the perspective of landscape ecology. The widespread application of geospatial techniques such as geographic information systems (GIS) and remote sensing (RS) in landscape ecology research, has demonstrated the necessity of assessing the progress of research in this field [17]. With the prevalence of Landsat imagery-based landscape indexes and application methods, land-use change maps constructed using multi-temporal data can be used to calculate landscape metrics and analyze the urban-to-rural gradient characteristics. Consequently, the spatiotemporal characteristics of landscape changes in metropolitan areas can be monitored, facilitating the investigation of the influence of urban expansion on both urban and rural landscapes $[18,19]$. Land-use change maps can also reflect the influence of land consolidation on local landscape patterns, which is vital for optimizing land consolidation models and accelerating the sustainable development of local communities [20,21]. The evolution of landscape patterns also leads to changes in the spatial and ecosystem structures of landscapes, which ultimately influences ecological security [22-24]. In previous studies related to agriculture and forestry, landscape pattern indexes and land-use change maps have not only reflected the influence of urbanization on cultivated land and forests, but have also been used to determine the service functions of cultivated lands and forests in urban ecosystems, thereby providing a reference for promoting sustainable development in urban and rural regions [25]. In this study, GIS and RS techniques were used for a case study that will help achieve sustainability in industrial rural areas, Southern Jiangsu, China. 
To reach this goal, we aimed to determine the actual influence of industrial rural development in Southern Jiangsu during 1981-2018 on local rural landscapes and the environments surrounding human settlements by using landscape pattern indexes. Four representative regions, i.e., the cities of Jiangyin, Zhangjiagang, Changshu, and Kunshan, were selected as study areas. In the County Economy Top 100 (2019) List of China by the government, Kunshan ranked No. 1, Jiangyin ranked No. 2, Zhangjiag ranked No. 3 and Changshu ranked No. 4, which means the four counties are the counties with the most developed economy in China. The study area is so peculiar, and representative of the most successful rural economic development model and one of the most representative models of the urbanization process in China since the economic reform of China. The economic achievement was based on industrialization, with damage to rural landscapes and natural environments, so it is necessary to sort out this typical kind of fast urbanization in regions driven by industry development. The specific aims of this case study are as follows: (1) Use GIS and RS techniques to establish land-use change maps from remotely sensed image data acquired by Landsat satellites during five periods in 1981, 1991, 2001, 2011, and 2018. (2) Calculate and analyze eight landscape pattern indexes at two different levels with ENVI and FRAGSTATS software. (3) Compare the landscape pattern evolution processes of the four study areas to analyze the landscape pattern evolution characteristics of industrial rural areas in Southern Jiangsu. (4) Explore factors driving landscape changes in this region. The results of this study concluded the landscape pattern and evolution dynamics of industrial rural areas in Southern Jiangsu, China, providing relevant fields with methods to investigate the evolution dynamic of the urban-rural industry during urbanization. It could also support and serve as a reference for other developing countries in Asia for sustainability of urban and rural development during industrialization.

The structure of this paper is organized as follows: In Section 2, we introduced and elaborated on the study area, data collection and pre-processing, as well as on the landscape pattern indexes chosen for this study; in Section 3, calculation results of the case study conducted in Southern Jiangsu were presented; in Section 4, experimental results and factors driving landscape pattern changes were discussed, and strategies for improving the landscape pattern and promoting the development of ecological environment and tourism were proposed, which is helpful to achieve sustainability; and in Section 5 , conclusions on the experimental results and future work were drawn.

\section{Materials and Methods}

\subsection{Study Area}

Jiangsu Province is divided into three major regions by the Yangtze and Huai Rivers, i.e., Southern, Central and Northern Jiangsu. Specifically, Southern Jiangsu contains the cities of Nanjing, Suzhou, Wuxi, Changzhou and Zhenjiang. According to statistics from 2017, the total population of Southern Jiangsu is approximately 33.4752 million, and the gross domestic product (GDP) of the region is approximately 5.0175 trillion yuan, accounting for $6 \%$ of China's GDP. The per-capita GDP of the region is 150,200 yuan, which is approximately three times the average per-capita GDP of the country. Industrial rural areas are rural locations dominated by industry, where the industrial output value accounts for the highest proportion of the total community assets, which have mainly been implemented in county-level cities under the jurisdiction of the three prefectural-level cities of Suzhou, Wuxi and Changzhou. Based on governmental statistics, we selected the following county-level cities as representative regions for this study, which have similar areas, similar population sizes, and respective GDPs of over 200 billion yuan: Jiangyin, Zhangiiagang, Changshu and Kunshan. The geographic coordinates of the spatial extent shown in Figure 1 are Jiangyin: $120^{\circ} 15^{\prime} 36^{\prime \prime} \mathrm{E}, 31^{\circ} 54^{\prime} 36^{\prime \prime} \mathrm{N}$; Zhangiiagang: $120^{\circ} 33^{\prime} 00^{\prime \prime} \mathrm{E}$, $31^{\circ} 52^{\prime} 12^{\prime \prime} \mathrm{N}$; Changshu: $120^{\circ} 44^{\prime} 24^{\prime \prime}$ E, $31^{\circ} 54^{\prime} 36^{\prime \prime} \mathrm{N}$; and Kunshan: $120^{\circ} 57^{\prime} 00^{\prime \prime}$ E, $31^{\circ} 23^{\prime} 24^{\prime \prime}$ N (Figure 1 and Table 1). 

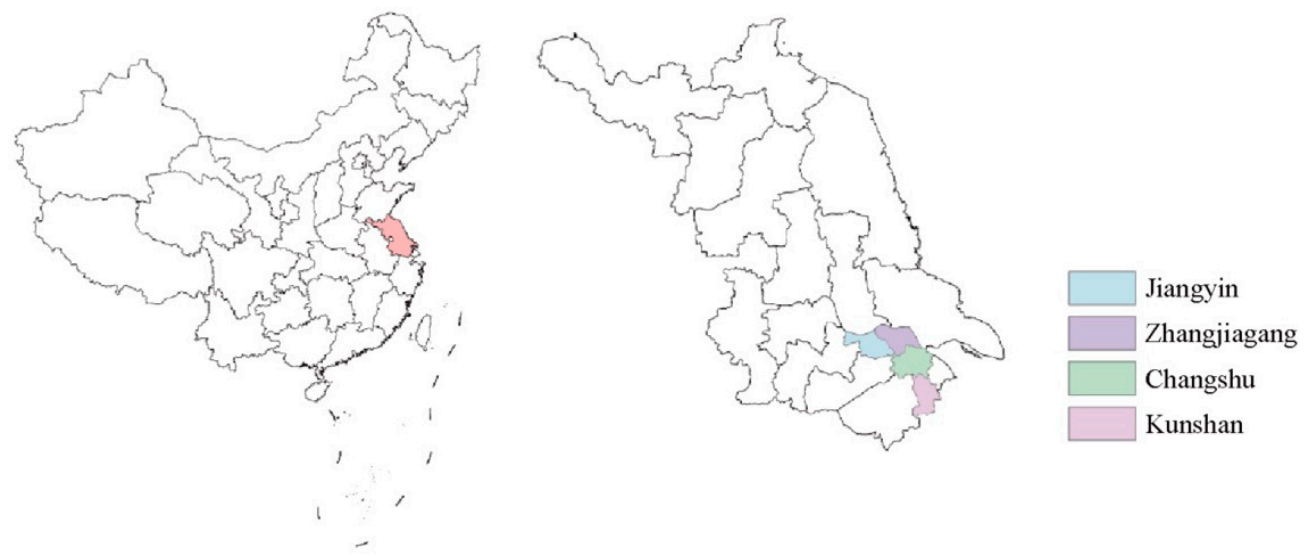

Figure 1. Study area.

Table 1. Statistical data of study area [26-29].

\begin{tabular}{|c|c|c|c|c|c|}
\hline Prefectural-Level & County-Level & Year & Population/Million & GDP/Billion & Area $/ \mathbf{k m}^{2}$ \\
\hline \multirow{4}{*}{ Wuxi } & \multirow{4}{*}{ Jiangyin } & 1990 & 1.10 & 3.67 & \multirow{4}{*}{987.53} \\
\hline & & 2001 & 1.15 & 36.50 & \\
\hline & & 2011 & 1.21 & 233.59 & \\
\hline & & 2017 & 1.25 & 348.83 & \\
\hline \multirow{12}{*}{ Suzhou } & \multirow{4}{*}{ Zhangjiagang } & 1990 & 0.83 & 2.78 & \multirow{4}{*}{999.00} \\
\hline & & 2001 & 0.85 & 30.68 & \\
\hline & & 2011 & 0.90 & 186.03 & \\
\hline & & 2017 & 0.92 & 260.61 & \\
\hline & \multirow{4}{*}{ Changshu } & 1990 & 1.03 & 3.63 & \multirow{4}{*}{1264.00} \\
\hline & & 2001 & 1.04 & 30.30 & \\
\hline & & 2011 & 1.06 & 171.05 & \\
\hline & & 2017 & 1.07 & 227.96 & \\
\hline & \multirow{4}{*}{ Kunshan } & 1990 & 0.56 & 2.01 & \multirow{4}{*}{927.68} \\
\hline & & 2001 & 0.60 & 23.08 & \\
\hline & & 2011 & 0.72 & 243.23 & \\
\hline & & 2017 & 0.86 & 352.03 & \\
\hline
\end{tabular}

\subsection{Data Collection and Pre-Processing}

Based on GIS and RS techniques, remotely sensed image data of the four subject cities acquired by Landsat satellites during five periods in 1981, 1991, 2001, 2011 and 2018 were utilized to extract the spatial distribution of various landscape categories. The satellite images were all captured in fall included the dates of 7 December 1981, 12 November 1991, 6 November 2001, 11 November 2011 and 24 December 2018. The resolution of the image in 1981 was $60 \mathrm{~m} \times 60 \mathrm{~m}$, whereas the others were all $30 \mathrm{~m} \times 30 \mathrm{~m}$. These were the highest resolution of images accessible to us. Resolution has been unified during land classification and resampling. FRAGSTATS 4.2 was used to calculate the landscape pattern indexes of each city during the different periods. By quantitatively and qualitatively analyzing the landscape pattern indexes, the influence of the rural industry on the evolution of landscape patterns was investigated. The remotely-sensed images were geometrically corrected in ERDAS 9.2 using the corresponding topographic maps as a reference. The images were then vectorized using ENVI 5.0. By following a human-computer interaction interpretation method, the landscapes were classified into four categories based on the characteristics of the studied areas: Construction land, cultivated land, green land and water bodies. Ground Truth ROI in ENVI 5.0 was used to assess the accuracy. And the kappa coefficients were all over 0.8648 , which was reliable. The classification maps obtained from visual interpretation were converted into the ArcGrid format using ArcGIS 10.2 and subsequently imported into FRAGSTATS 4.2 for calculating the landscape pattern indexes. 


\subsection{Landscape Pattern Indexes}

In this study, we utilized landscape pattern indexes, including patch-class-level and landscape-level indexes, to portray the characteristics of the spatiotemporal changes in the various landscape categories. The patch-class-level indexes included the percentage of landscape (PLAND), number of patches (NP), patch density (PD) and largest patch index (LPI), while the landscape-level indexes included the contagion index (CONTAG), splitting index (SPLIT), Shannon's diversity index (SHDI) and Shannon's evenness index (SHEI). The evolution process of the landscape patterns was determined by investigating the basic characteristics, morphological changes and spatiotemporal evolution of the various landscape categories, as shown in Table 2 . The annual changing ratio of landscape pattern indexes in the patch-class level was calculated to show the annual change of four kinds of lands clearly. The formula was ((year2-year1)/year1) $\times 100 \%$.

Table 2. Landscape pattern index used in this study.

\begin{tabular}{|c|c|c|}
\hline Name & Calculation Formula & Notes \\
\hline $\begin{array}{l}\text { Proportion of landscape } \\
\text { types (PLAND) [30] }\end{array}$ & PLAND $=P_{i}=\frac{\sum_{j=1}^{n} a_{i j}}{A}(100)$ & $\begin{array}{l}a_{i j} \text { represents the area of patches } \\
\text { numbered ij, and } A \text { represents the total } \\
\text { area of all patches. }\end{array}$ \\
\hline $\begin{array}{l}\text { Number of patches } \\
\text { (NP) [30] }\end{array}$ & $N P=n_{i}$ & $\begin{array}{l}n_{i} \text { represents the total number of patches } \\
\text { contained in type I of the entire landscape. }\end{array}$ \\
\hline Patch density (PD) [30] & $P D=\frac{1}{A} \sum_{j=1}^{M} N_{i}$ & $\begin{array}{l}\text { A represents the total area of all patches } \\
M \text { represents the total number of } \\
\text { landscape element types at a spatial } \\
\text { resolution within the scope of the study }\end{array}$ \\
\hline $\begin{array}{l}\text { Largest patch index } \\
\text { (LPI) [30] }\end{array}$ & $L P I=\frac{\max \left(a_{i j}\right)}{A}(100)$ & $\begin{array}{l}a_{i j} \text { represents the area of patches } \\
\text { numbered ij, and } A \text { represents the total } \\
\text { area of all patches. }\end{array}$ \\
\hline $\begin{array}{l}\text { Contagion index } \\
\text { (CONTAG) [30] }\end{array}$ & {$\left[\begin{array}{c}\text { CONTAG }= \\
\left.1+\frac{\sum_{i=1}^{m} \sum_{k=1}^{m}\left[\left(P_{i}\right) \frac{s_{i k}}{\sum_{k=1}^{m} s_{i k}}\right]\left[\ln \left(P_{i}\right) \frac{s_{i k}}{\sum_{k=1}^{m_{i k}}}\right]}{2 \ln (m)}\right](100)\end{array}\right.$} & $\begin{array}{l}P_{i} \text { represents the percentage of area } \\
\text { occupied by type I; } g_{i k} \text { represents the } \\
\text { number of IK adjacent to the plaque type. } \\
M \text { represents the total number of patch } \\
\text { types in the landscape }\end{array}$ \\
\hline Splitting Index (SPLIT) [30] & $C_{i}=\frac{N_{i}}{A_{i}}$ & $\begin{array}{l}N_{i} \text { represents the number of patches. } \\
A_{i} \text { represents the total area of all patches. }\end{array}$ \\
\hline $\begin{array}{l}\text { Shannon's diversity index } \\
\text { (SHDI) [30] }\end{array}$ & $\begin{array}{l}\text { SHDI }=-\sum_{k=1}^{m} P_{k} \ln \left(P_{k}\right) \\
\quad S H D I_{\max }=\ln (m)\end{array}$ & $\begin{array}{l}P_{k} \text { represents the probability of patch type } \\
\mathrm{K} \text { appearing in landscape. } \\
m \text { represents the total number of patch } \\
\text { types in the landscape. }\end{array}$ \\
\hline $\begin{array}{l}\text { Shannon's evenness index } \\
\text { (SHEI) [30] }\end{array}$ & $S H E I=\frac{S H D I}{S H D I_{\max }}=\frac{-\sum_{k=1}^{m} P_{k} \ln \left(P_{k}\right)}{\ln (m)}$ & $\begin{array}{l}P_{k} \text { represents the probability of patch type } \\
\text { K appearing in landscape. } \\
m \text { represents the total number of patch } \\
\text { types in the landscape. }\end{array}$ \\
\hline
\end{tabular}

\section{Results}

\subsection{Land Classification Results}

The characteristics of the land-use categories of the four study areas during the five periods described above extracted using ENVI 5.0 are shown in Figure 2, as well as the patch-class-level and landscape-level landscape pattern indexes of each study area calculated using FRAGSTATS 4.2. 


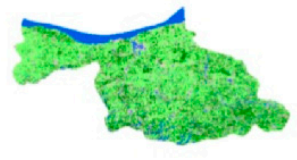

Jiangyin 1981

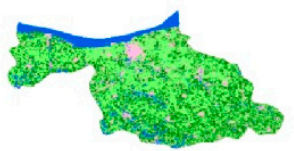

Jiangyin 1991

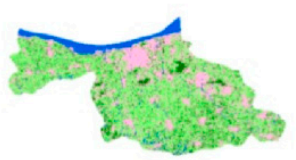

Jiangyin 2001

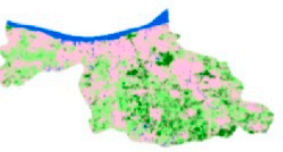

Jiangyin 2011

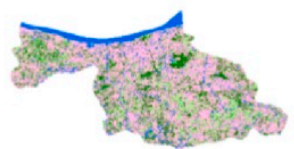

Jiangyin 2018

Legend

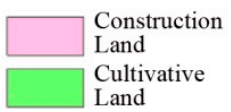

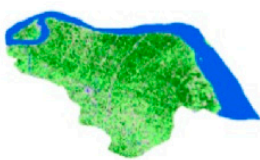

Zhangjiagang 1981

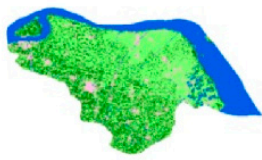

Zhangjiagang 1991

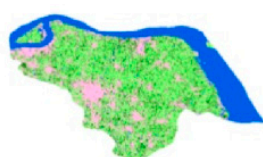

Zhangjiagang 2001

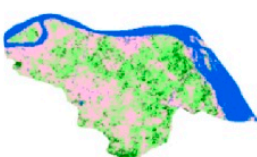

Zhangjiagang 2011

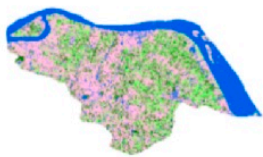

Zhangjiagang 2018

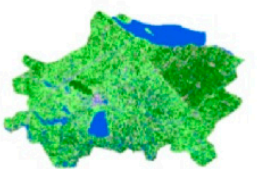

Changshu 1981

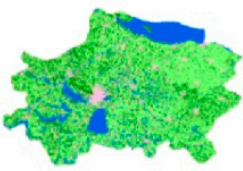

Changshu 1991

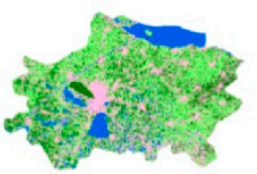

Changshu 2001

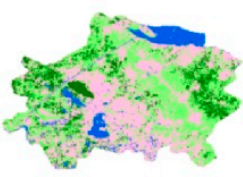

Changshu 2011

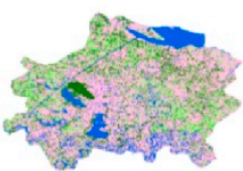

Changshu 2018

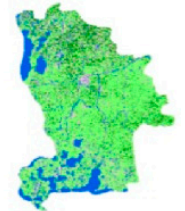

Kunshan 1981

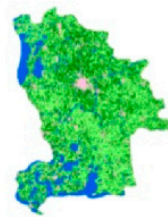

Kunshan 1991

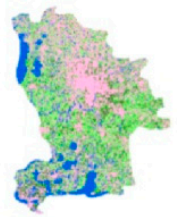

Kunshan 2001

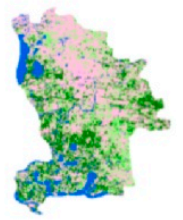

Kunshan 2011

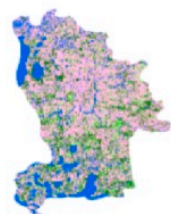

Kunshan 2018

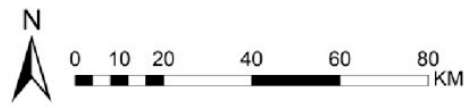

Figure 2. Land classification results.

\subsection{Patch-Class-Level Landscape Pattern Results}

Tables 3-10 show the results of the calculation and analysis of the patch-class-level landscape pattern indexes selected in this study (PLAND, NP, PD and LPI).

In all four study areas, the PLAND of construction land exhibited a rapid, continuous increase. The annual increase during 1981-2018 exceeded $400 \%$ in all areas, with the highest annual increase of $637.03 \%$ being observed in Changshu City. In contrast, the PLAND of cultivated land in all four study areas decreased continuously over time. Figure 3 shows that the annual decrease during 1981-2018 exceeded 50\% in all areas, with Kunshan City exhibiting the highest annual decrease of $80.17 \%$. The PLAND of green land decreased in the cities of Jiangyin, Zhangiiagang and Changshu, while that in Kunshan increased. No significant changes in the PLAND of water bodies were observed in all four study areas. 
Table 3. Calculation results from the patch-class-level in Jiangyin.

\begin{tabular}{|c|c|c|c|c|c|c|}
\hline City & Category & Year & PLAND & NP & PD & LPI \\
\hline \multirow{20}{*}{ Jiangyin } & \multirow{5}{*}{$\begin{array}{c}\text { Construction } \\
\text { Land }\end{array}$} & 1981 & 9.59 & 2186.00 & 2.24 & 0.20 \\
\hline & & 1991 & 17.56 & 1269.00 & 1.30 & 1.23 \\
\hline & & 2001 & 28.90 & 1897.00 & 1.95 & 6.42 \\
\hline & & 2011 & 46.76 & 923.00 & 0.95 & 32.83 \\
\hline & & 2018 & 57.61 & 228.00 & 0.23 & 56.50 \\
\hline & \multirow{5}{*}{$\begin{array}{l}\text { Green } \\
\text { Land }\end{array}$} & 1981 & 23.27 & 2201.00 & 2.26 & 1.97 \\
\hline & & 1991 & 29.23 & 797.00 & 0.82 & 1.23 \\
\hline & & 2001 & 23.04 & 2836.00 & 2.91 & 0.44 \\
\hline & & 2011 & 10.11 & 956.00 & 0.98 & 0.64 \\
\hline & & 2018 & 18.71 & 2234.00 & 2.29 & 0.95 \\
\hline & \multirow{5}{*}{$\begin{array}{l}\text { Water } \\
\text { Bodies }\end{array}$} & 1981 & 9.18 & 1056.00 & 1.08 & 5.31 \\
\hline & & 1991 & 10.75 & 873.00 & 0.90 & 6.09 \\
\hline & & 2001 & 10.97 & 480.00 & 0.49 & 5.20 \\
\hline & & 2011 & 10.60 & 315.00 & 0.32 & 4.45 \\
\hline & & 2018 & 10.71 & 1441.00 & 1.48 & 4.71 \\
\hline & \multirow{5}{*}{$\begin{array}{l}\text { Cultivated } \\
\text { Land }\end{array}$} & 1981 & 57.96 & 245.00 & 0.25 & 55.75 \\
\hline & & 1991 & 42.46 & 690.00 & 0.71 & 30.91 \\
\hline & & 2001 & 37.09 & 359.00 & 0.37 & 47.25 \\
\hline & & 2011 & 32.53 & 1010.00 & 1.04 & 19.91 \\
\hline & & 2018 & 12.98 & 2365.00 & 2.42 & 0.52 \\
\hline
\end{tabular}

Table 4. Calculation results from the patch-class-level in Zhangjiagang.

\begin{tabular}{|c|c|c|c|c|c|c|}
\hline City & Category & Year & PLAND & NP & PD & LPI \\
\hline \multirow{20}{*}{ Zhangjiagang } & \multirow{5}{*}{$\begin{array}{l}\text { Construction } \\
\text { Land }\end{array}$} & 1981 & 8.86 & 1615.00 & 1.61 & 0.31 \\
\hline & & 1991 & 10.33 & 884.00 & 0.45 & 0.48 \\
\hline & & 2001 & 21.24 & 1446.00 & 1.44 & 4.54 \\
\hline & & 2011 & 36.69 & 739.00 & 0.73 & 22.18 \\
\hline & & 2018 & 45.40 & 411.00 & 0.41 & 38.06 \\
\hline & \multirow{5}{*}{$\begin{array}{l}\text { Green } \\
\text { Land }\end{array}$} & 1981 & 29.08 & 1140.00 & 1.13 & 16.94 \\
\hline & & 1991 & 15.98 & 1250.00 & 1.24 & 2.34 \\
\hline & & 2001 & 12.71 & 2414.00 & 2.40 & 0.11 \\
\hline & & 2011 & 6.98 & 869.00 & 0.86 & 0.54 \\
\hline & & 2018 & 13.68 & 2033.00 & 2.02 & 0.17 \\
\hline & \multirow{5}{*}{$\begin{array}{l}\text { Water } \\
\text { Bodies }\end{array}$} & 1981 & 21.80 & 592.00 & 0.59 & 20.16 \\
\hline & & 1991 & 22.69 & 459.00 & 0.46 & 20.55 \\
\hline & & 2001 & 21.03 & 281.00 & 0.28 & 20.09 \\
\hline & & 2011 & 21.22 & 182.00 & 0.18 & 17.53 \\
\hline & & 2018 & 21.09 & 943.00 & 0.94 & 17.40 \\
\hline & \multirow{5}{*}{$\begin{array}{l}\text { Cultivated } \\
\text { Land }\end{array}$} & 1981 & 40.25 & 456.00 & 0.45 & 28.14 \\
\hline & & 1991 & 51.01 & 161.00 & 0.16 & 49.08 \\
\hline & & 2001 & 45.02 & 226.00 & 0.22 & 41.77 \\
\hline & & 2011 & 35.11 & 608.00 & 0.60 & 31.55 \\
\hline & & 2018 & 19.83 & 1430.00 & 1.42 & 1.92 \\
\hline
\end{tabular}


Table 5. Calculation results from the patch-class-level in Changshu.

\begin{tabular}{|c|c|c|c|c|c|c|}
\hline City & Category & Year & PLAND & NP & PD & LPI \\
\hline \multirow{20}{*}{ Changshu } & \multirow{5}{*}{$\begin{array}{c}\text { Construction } \\
\text { Land }\end{array}$} & 1981 & 7.15 & 1987.00 & 1.64 & 0.28 \\
\hline & & 1991 & 16.11 & 1258.00 & 1.04 & 0.83 \\
\hline & & 2001 & 24.26 & 2110.00 & 1.74 & 4.72 \\
\hline & & 2011 & 36.69 & 964.00 & 0.80 & 25.08 \\
\hline & & 2018 & 52.70 & 412.00 & 0.34 & 48.41 \\
\hline & \multirow{5}{*}{$\begin{array}{l}\text { Green } \\
\text { Land }\end{array}$} & 1981 & 34.12 & 1655.00 & 1.37 & 12.42 \\
\hline & & 1991 & 19.98 & 2627.00 & 0.86 & 0.64 \\
\hline & & 2001 & 22.79 & 2293.00 & 0.70 & 2.07 \\
\hline & & 2011 & 13.68 & 996.00 & 0.82 & 2.83 \\
\hline & & 2018 & 15.29 & 2510.00 & 2.07 & 1.13 \\
\hline & \multirow{5}{*}{$\begin{array}{l}\text { Water } \\
\text { Bodies }\end{array}$} & 1981 & 13.36 & 1349.00 & 1.11 & 5.52 \\
\hline & & 1991 & 12.88 & 1038.00 & 0.86 & 5.30 \\
\hline & & 2001 & 11.61 & 850.00 & 0.70 & 5.02 \\
\hline & & 2011 & 12.79 & 484.00 & 0.40 & 3.88 \\
\hline & & 2018 & 13.62 & 1414.00 & 1.17 & 3.84 \\
\hline & \multirow{5}{*}{$\begin{array}{l}\text { Cultivated } \\
\text { Land }\end{array}$} & 1981 & 45.36 & 811.00 & 0.67 & 37.50 \\
\hline & & 1991 & 51.04 & 143.00 & 0.12 & 59.43 \\
\hline & & 2001 & 41.34 & 862.00 & 0.71 & 28.91 \\
\hline & & 2011 & 36.85 & 919.00 & 0.76 & 26.93 \\
\hline & & 2018 & 18.40 & 2008.00 & 1.66 & 0.97 \\
\hline
\end{tabular}

Table 6. Calculation results from the patch-class-level in Kunshan.

\begin{tabular}{|c|c|c|c|c|c|c|}
\hline City & Category & Year & PLAND & NP & PD & LPI \\
\hline \multirow{20}{*}{ Kunshan } & \multirow{5}{*}{$\begin{array}{l}\text { Construction } \\
\text { Land }\end{array}$} & 1981 & 9.87 & 2475.00 & 2.60 & 0.25 \\
\hline & & 1991 & 15.37 & 862.00 & 0.90 & 0.66 \\
\hline & & 2001 & 42.79 & 1118.00 & 1.17 & 24.39 \\
\hline & & 2011 & 41.40 & 932.00 & 0.98 & 26.52 \\
\hline & & 2018 & 53.96 & 312.00 & 0.33 & 50.99 \\
\hline & \multirow{5}{*}{$\begin{array}{l}\text { Green } \\
\text { Land }\end{array}$} & 1981 & 12.03 & 2201.00 & 2.31 & 0.70 \\
\hline & & 1991 & 30.47 & 1965.00 & 2.06 & 6.04 \\
\hline & & 2001 & 12.07 & 2787.00 & 2.92 & 0.25 \\
\hline & & 2011 & 22.13 & 1236.00 & 1.30 & 3.60 \\
\hline & & 2018 & 18.87 & 2243.00 & 2.35 & 0.69 \\
\hline & \multirow{5}{*}{$\begin{array}{l}\text { Water } \\
\text { Bodies }\end{array}$} & 1981 & 15.58 & 1510.00 & 1.58 & 3.48 \\
\hline & & 1991 & 13.43 & 944.00 & 0.99 & 3.02 \\
\hline & & 2001 & 13.69 & 1472.00 & 1.54 & 1.86 \\
\hline & & 2011 & 13.62 & 773.00 & 0.81 & 1.87 \\
\hline & & 2018 & 14.77 & 1727.00 & 1.81 & 1.92 \\
\hline & \multirow{5}{*}{$\begin{array}{l}\text { Cultivated } \\
\text { Land }\end{array}$} & 1981 & 62.52 & 190.00 & 0.20 & 60.98 \\
\hline & & 1991 & 40.72 & 352.00 & 0.37 & 46.08 \\
\hline & & 2001 & 31.45 & 1270.00 & 1.33 & 5.27 \\
\hline & & 2011 & 22.85 & 1942.00 & 2.04 & 1.82 \\
\hline & & 2018 & 12.39 & 2086.00 & 2.19 & 0.20 \\
\hline
\end{tabular}


Table 7. Changes of landscape indexes in Jiangyin.

\begin{tabular}{|c|c|c|c|c|c|c|}
\hline City & Category & Year & PLAND & NP & PD & LPI \\
\hline \multirow{20}{*}{ Jiangyin } & \multirow{5}{*}{$\begin{array}{c}\text { Construction } \\
\text { Land }\end{array}$} & 1981-1991 & $83.22 \%$ & $-41.95 \%$ & $-41.95 \%$ & $518.57 \%$ \\
\hline & & $1991-2001$ & $64.55 \%$ & $49.49 \%$ & $49.49 \%$ & $423.80 \%$ \\
\hline & & 2001-2011 & $61.80 \%$ & $-51.34 \%$ & $-51.34 \%$ & $411.29 \%$ \\
\hline & & 2011-2018 & $23.19 \%$ & $-75.30 \%$ & $-75.30 \%$ & $72.09 \%$ \\
\hline & & 1981-2018 & $500.94 \%$ & $-89.57 \%$ & $-89.57 \%$ & $28,407.87 \%$ \\
\hline & \multirow{5}{*}{$\begin{array}{l}\text { Green } \\
\text { Land }\end{array}$} & 1981-1991 & $32.51 \%$ & $73.44 \%$ & $73.45 \%$ & $60.33 \%$ \\
\hline & & 1991-2001 & $1.13 \%$ & $-57.99 \%$ & $-57.98 \%$ & $-80.91 \%$ \\
\hline & & 2001-2011 & $-50.73 \%$ & $207.26 \%$ & $207.25 \%$ & $-98.65 \%$ \\
\hline & & 2011-2018 & $-82.45 \%$ & $319.30 \%$ & $319.25 \%$ & $-98.86 \%$ \\
\hline & & $1981-2018$ & $-19.62 \%$ & $1.50 \%$ & $1.50 \%$ & $-51.44 \%$ \\
\hline & \multirow{5}{*}{ Water Bodies } & 1981-1991 & $17.11 \%$ & $-17.33 \%$ & $-17.33 \%$ & $14.70 \%$ \\
\hline & & $1991-2001$ & $2.07 \%$ & $-45.02 \%$ & $-45.01 \%$ & $-14.63 \%$ \\
\hline & & 2001-2011 & $-3.45 \%$ & $-34.38 \%$ & $-34.38 \%$ & $-14.41 \%$ \\
\hline & & 2011-2018 & $1.03 \%$ & $357.46 \%$ & $357.43 \%$ & $5.98 \%$ \\
\hline & & 1981-2018 & $16.61 \%$ & $36.46 \%$ & $36.46 \%$ & $-11.17 \%$ \\
\hline & \multirow{5}{*}{ Cultivated } & 1981-1991 & $26.71 \%$ & $-64.69 \%$ & $-64.70 \%$ & $74.39 \%$ \\
\hline & & 1991-2001 & $-26.75 \%$ & $181.63 \%$ & $181.65 \%$ & $-44.55 \%$ \\
\hline & & 2001-2011 & $-12.65 \%$ & $-47.97 \%$ & $-47.97 \%$ & $52.85 \%$ \\
\hline & & 2011-2018 & $-12.28 \%$ & $181.34 \%$ & $181.34 \%$ & $-57.87 \%$ \\
\hline & & $1981-2018$ & $-77.60 \%$ & $865.31 \%$ & $865.33 \%$ & $-99.07 \%$ \\
\hline
\end{tabular}

Table 8. Changes of landscape indexes in Zhangjiagang.

\begin{tabular}{|c|c|c|c|c|c|c|}
\hline City & Category & Year & PLAND & NP & PD & LPI \\
\hline \multirow{20}{*}{ Zhangjiagang } & \multirow{5}{*}{$\begin{array}{l}\text { Construction } \\
\text { Land }\end{array}$} & 1981-1991 & $16.49 \%$ & $-45.26 \%$ & $-71.76 \%$ & $54.54 \%$ \\
\hline & & 1991-2001 & $105.65 \%$ & $63.57 \%$ & $217.10 \%$ & $844.20 \%$ \\
\hline & & 2001-2011 & $72.75 \%$ & $-48.89 \%$ & $-48.89 \%$ & $389.01 \%$ \\
\hline & & 2011-2018 & $23.75 \%$ & $-44.38 \%$ & $-44.38 \%$ & $71.64 \%$ \\
\hline & & 1981-2018 & $412.16 \%$ & $-74.55 \%$ & $-74.55 \%$ & $12,147.14 \%$ \\
\hline & \multirow{5}{*}{$\begin{array}{l}\text { Green } \\
\text { Land }\end{array}$} & 1981-1991 & $-45.06 \%$ & $9.65 \%$ & $9.65 \%$ & $-86.21 \%$ \\
\hline & & 1991-2001 & $-20.41 \%$ & $93.12 \%$ & $93.12 \%$ & $-95.25 \%$ \\
\hline & & 2001-2011 & $-45.08 \%$ & $-64.00 \%$ & $-64.00 \%$ & $389.10 \%$ \\
\hline & & 2011-2018 & $95.95 \%$ & $133.95 \%$ & $133.95 \%$ & $-69.15 \%$ \\
\hline & & 1981-2018 & $-52.95 \%$ & $78.33 \%$ & $78.34 \%$ & $-99.01 \%$ \\
\hline & \multirow{5}{*}{ Water Bodies } & 1981-1991 & $4.09 \%$ & $-22.47 \%$ & $-22.46 \%$ & $1.93 \%$ \\
\hline & & 1991-2001 & $-7.33 \%$ & $-38.78 \%$ & $-38.79 \%$ & $-2.21 \%$ \\
\hline & & 2001-2011 & $0.93 \%$ & $-35.23 \%$ & $-35.23 \%$ & $-12.77 \%$ \\
\hline & & 2011-2018 & $-0.63 \%$ & $418.13 \%$ & $418.19 \%$ & $-0.70 \%$ \\
\hline & & 1981-2018 & $-3.26 \%$ & $59.29 \%$ & $59.29 \%$ & $-13.66 \%$ \\
\hline & \multirow{5}{*}{$\begin{array}{l}\text { Cultivated } \\
\text { Land }\end{array}$} & 1981-1991 & $26.71 \%$ & $-64.69 \%$ & $-64.70 \%$ & $74.39 \%$ \\
\hline & & 1991-2001 & $-11.74 \%$ & $40.37 \%$ & $40.44 \%$ & $-14.89 \%$ \\
\hline & & 2001-2011 & $-22.02 \%$ & $169.03 \%$ & $168.98 \%$ & $-24.46 \%$ \\
\hline & & 2011-2018 & $-43.52 \%$ & $135.20 \%$ & $135.19 \%$ & $-93.92 \%$ \\
\hline & & 1981-2018 & $-50.74 \%$ & $213.60 \%$ & $213.59 \%$ & $-93.18 \%$ \\
\hline
\end{tabular}


Table 9. Changes of landscape indexes in Changshu.

\begin{tabular}{|c|c|c|c|c|c|c|}
\hline City & Category & Year & PLAND & NP & PD & LPI \\
\hline \multirow{20}{*}{ Changshu } & \multirow{5}{*}{$\begin{array}{l}\text { Construction } \\
\text { Land }\end{array}$} & 1981-1991 & $125.25 \%$ & $-36.69 \%$ & $-36.69 \%$ & $193.36 \%$ \\
\hline & & 1991-2001 & $50.61 \%$ & $67.73 \%$ & $67.73 \%$ & $465.91 \%$ \\
\hline & & 2001-2011 & $51.24 \%$ & $-54.31 \%$ & $-54.31 \%$ & $430.88 \%$ \\
\hline & & 2011-2018 & $43.65 \%$ & $-57.26 \%$ & $-57.26 \%$ & $92.99 \%$ \\
\hline & & 1981-2018 & $637.03 \%$ & $-79.27 \%$ & $-79.27 \%$ & $16,908.96 \%$ \\
\hline & \multirow{5}{*}{$\begin{array}{l}\text { Green } \\
\text { Land }\end{array}$} & 1981-1991 & $-41.45 \%$ & $58.73 \%$ & $-37.28 \%$ & $-94.87 \%$ \\
\hline & & 1991-2001 & $14.09 \%$ & $-12.71 \%$ & $-18.11 \%$ & $225.15 \%$ \\
\hline & & $2001-2011$ & $-40.01 \%$ & $-56.56 \%$ & $17.17 \%$ & $36.67 \%$ \\
\hline & & 2011-2018 & $11.78 \%$ & $152.01 \%$ & $152.01 \%$ & $-60.26 \%$ \\
\hline & & 1981-2018 & $-55.20 \%$ & $51.66 \%$ & $51.66 \%$ & $-90.94 \%$ \\
\hline & \multirow{5}{*}{$\begin{array}{l}\text { Water } \\
\text { Bodies }\end{array}$} & 1981-1991 & $-3.65 \%$ & $-23.05 \%$ & $-23.05 \%$ & $-3.86 \%$ \\
\hline & & 1991-2001 & $-9.87 \%$ & $-18.11 \%$ & $-18.11 \%$ & $-5.29 \%$ \\
\hline & & 2001-2011 & $10.20 \%$ & $-43.06 \%$ & $-43.06 \%$ & $-22.78 \%$ \\
\hline & & 2011-2018 & $6.47 \%$ & $192.15 \%$ & $192.17 \%$ & $-0.97 \%$ \\
\hline & & 1981-2018 & $1.89 \%$ & $4.82 \%$ & $4.82 \%$ & $-30.37 \%$ \\
\hline & \multirow{5}{*}{$\begin{array}{l}\text { Cultivated } \\
\text { Land }\end{array}$} & 1981-1991 & $12.51 \%$ & $-82.37 \%$ & $-82.37 \%$ & $58.49 \%$ \\
\hline & & 1991-2001 & $-19.00 \%$ & $502.80 \%$ & $502.96 \%$ & $-51.36 \%$ \\
\hline & & 2001-2011 & $-10.87 \%$ & $6.61 \%$ & $6.61 \%$ & $-6.83 \%$ \\
\hline & & 2011-2018 & $-50.08 \%$ & $118.50 \%$ & $118.49 \%$ & $-96.40 \%$ \\
\hline & & 1981-2018 & $-59.45 \%$ & $147.60 \%$ & $147.62 \%$ & $-97.41 \%$ \\
\hline
\end{tabular}

Table 10. Changes of landscape indexes in Kunshan.

\begin{tabular}{|c|c|c|c|c|c|c|}
\hline City & Category & Year & PLAND & NP & PD & LPI \\
\hline \multirow{20}{*}{ Kunshan } & \multirow{5}{*}{$\begin{array}{l}\text { Construction } \\
\text { Land }\end{array}$} & 1981-1991 & $55.85 \%$ & $-65.17 \%$ & $-65.17 \%$ & $159.74 \%$ \\
\hline & & 1991-2001 & $178.30 \%$ & $29.70 \%$ & $29.71 \%$ & $3587.65 \%$ \\
\hline & & 2001-2011 & $-3.23 \%$ & $-16.64 \%$ & $-16.64 \%$ & $8.73 \%$ \\
\hline & & 2011-2018 & $30.34 \%$ & $-66.52 \%$ & $-66.52 \%$ & $92.29 \%$ \\
\hline & & 1981-2018 & $447.01 \%$ & $-87.39 \%$ & $-87.39 \%$ & $19,926.20 \%$ \\
\hline & \multirow{5}{*}{$\begin{array}{l}\text { Green } \\
\text { Land }\end{array}$} & 1981-1991 & $153.33 \%$ & $-10.72 \%$ & $-10.72 \%$ & $761.93 \%$ \\
\hline & & 1991-2001 & $-60.39 \%$ & $41.83 \%$ & $41.83 \%$ & $-95.84 \%$ \\
\hline & & 2001-2011 & $83.36 \%$ & $-55.65 \%$ & $-55.65 \%$ & $1334.49 \%$ \\
\hline & & 2011-2018 & $-14.74 \%$ & $81.47 \%$ & $81.48 \%$ & $-80.76 \%$ \\
\hline & & 1981-2018 & $56.86 \%$ & $1.91 \%$ & $1.91 \%$ & $-1.00 \%$ \\
\hline & \multirow{5}{*}{$\begin{array}{l}\text { Water } \\
\text { Bodies }\end{array}$} & 1981-1991 & $-13.84 \%$ & $-37.48 \%$ & $-37.49 \%$ & $-13.26 \%$ \\
\hline & & 1991-2001 & $1.96 \%$ & $55.93 \%$ & $55.94 \%$ & $-38.29 \%$ \\
\hline & & 2001-2011 & $-0.52 \%$ & $-47.49 \%$ & $-47.48 \%$ & $0.28 \%$ \\
\hline & & 2011-2018 & $8.46 \%$ & $123.42 \%$ & $123.41 \%$ & $2.74 \%$ \\
\hline & & 1981-2018 & $-5.21 \%$ & $14.37 \%$ & $14.37 \%$ & $-44.84 \%$ \\
\hline & \multirow{5}{*}{$\begin{array}{l}\text { Cultivated } \\
\text { Land }\end{array}$} & 1981-1991 & $-34.86 \%$ & $85.26 \%$ & $85.25 \%$ & $-24.43 \%$ \\
\hline & & 1991-2001 & $-22.77 \%$ & $260.80 \%$ & $260.83 \%$ & $-88.57 \%$ \\
\hline & & 2001-2011 & $-27.37 \%$ & $52.91 \%$ & $52.91 \%$ & $-65.41 \%$ \\
\hline & & 2011-2018 & $-45.74 \%$ & $7.42 \%$ & $7.42 \%$ & $-89.22 \%$ \\
\hline & & 1981-2018 & $-80.17 \%$ & $997.89 \%$ & $997.94 \%$ & $-99.68 \%$ \\
\hline
\end{tabular}




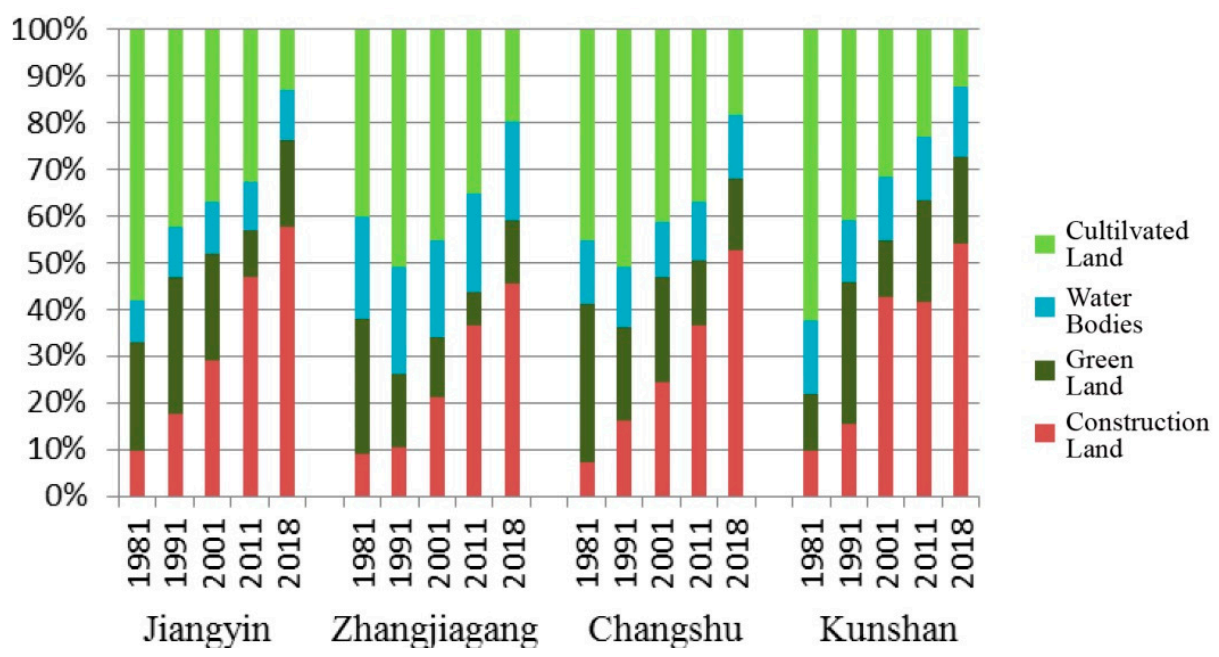

Figure 3. Changes in percentage of landscape (PLAND).

NP and PD reflect the degree of fragmentation of a certain patch class. Figures 4 and 5 show that the NP and PD of construction land decreased in all four study areas, while those of cultivated land, green land and water bodies increased. Therefore, the degree of fragmentation of construction land decreased in all study areas, while that of cultivated land, green land and water bodies increased.

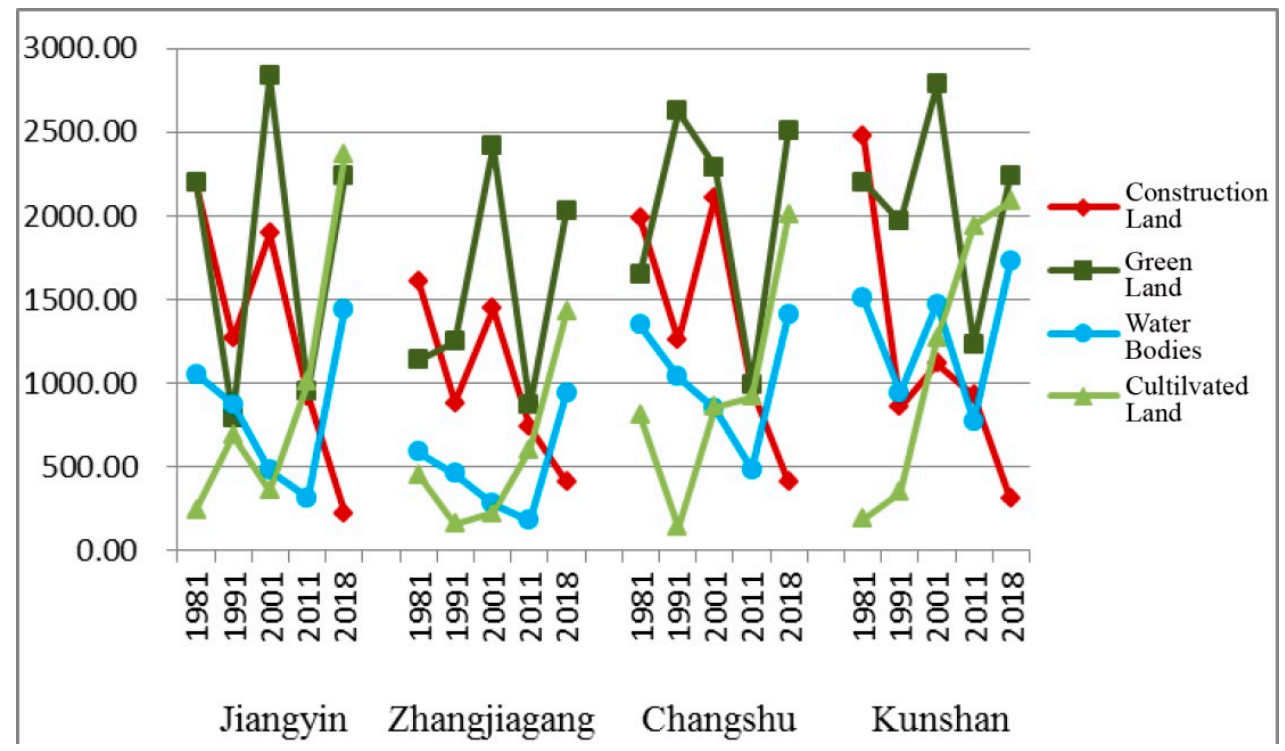

Figure 4. Changes in number of patches (NP).

LPI is a measure of landscape dominance, and the dynamic characteristics of the calculated LPI values indicate that the dominant landscape type in Jiangyin, Zhangjiagang and Changshu was cultivated land during 1981-2011, and construction land during 2011-2018. Additionally, the LPI of water bodies in all four study areas gradually decreased during 1981-2018, i.e., the landscape dominance of the "water body" patch class decreased continuously. The dominant landscape category in Kunshan City was cultivated land during 1981-2001, and construction land during 2001-2018 (Figure 6). 


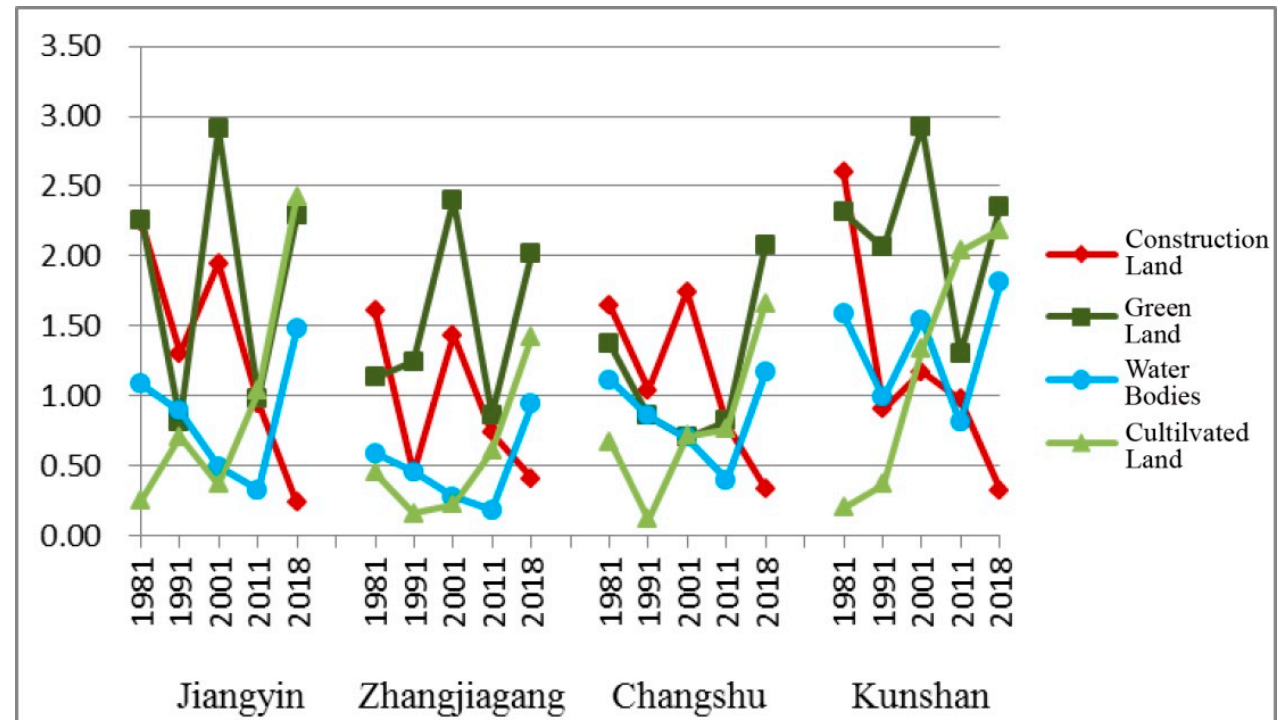

Figure 5. Changes in patch density (PD).

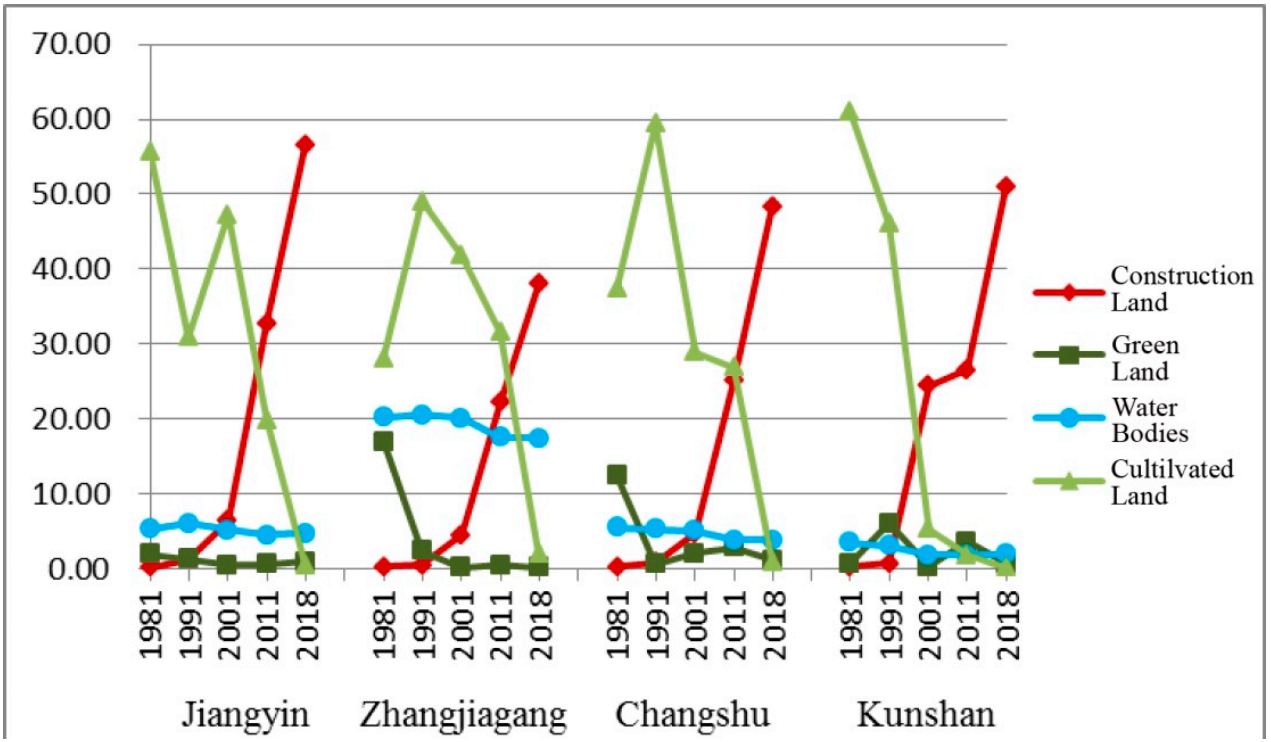

Figure 6. Changes in largest patch index (LPI).

In all four study areas, construction land continuously encroached on green land, exhibiting trends of expansion, centralization and the continuous consolidation of small patches into large patches during its evolution and ultimately replaced cultivated land as the dominant landscape category. Concurrently, the green and cultivated land patches, which are high-quality landscape resources, were continuously segmented into a larger number of small patches, which led to fragmentation during the evolution of green and cultivated land. Additionally, the water bodies in the four study areas should receive special attention; although the changes in PLAND during 1981-2018 were not significant, the dynamic changes in NP, PD and LPI indicate that the water bodies also faced fragmentation and reductions in dominance. Therefore, the effects of human disturbance on water bodies should not be neglected, even though they are less severe than those experienced by green and cultivated land.

\subsection{Landscape-Level Landscape Pattern Results}

Table 11 shows the results of the calculation and analysis of the landscape-level landscape pattern indexes, including CONTAG, SPLIT, SHDI and SHEI. 
Table 11. Calculation results from the landscape-level.

\begin{tabular}{|c|c|c|c|c|c|}
\hline & Year & CONTAG & SPLIT & SHDI & SHEI \\
\hline \multirow{5}{*}{ Jiangyin } & 1981 & 25.93 & 3.18 & 1.10 & 0.79 \\
\hline & 1991 & 22.88 & 8.02 & 1.17 & 0.84 \\
\hline & 2001 & 24.24 & 4.33 & 1.15 & 0.83 \\
\hline & 2011 & 28.84 & 6.53 & 1.12 & 0.81 \\
\hline & 2018 & 22.25 & 3.11 & 1.14 & 0.82 \\
\hline \multirow{5}{*}{ Zhangjiagang } & 1981 & 22.75 & 2.56 & 1.27 & 0.92 \\
\hline & 1991 & 31.06 & 3.52 & 1.16 & 0.84 \\
\hline & 2001 & 24.29 & 4.59 & 1.28 & 0.92 \\
\hline & 2011 & 28.37 & 5.50 & 1.23 & 0.89 \\
\hline & 2018 & 18.72 & 5.67 & 1.28 & 0.92 \\
\hline \multirow{5}{*}{ Changshu } & 1981 & 20.15 & 2.25 & 1.18 & 0.85 \\
\hline & 1991 & 29.92 & 2.80 & 1.06 & 0.76 \\
\hline & 2001 & 13.46 & 11.13 & 1.30 & 0.93 \\
\hline & 2011 & 24.21 & 16.98 & 1.20 & 0.87 \\
\hline & 2018 & 17.31 & 14.23 & 1.21 & 0.87 \\
\hline \multirow{5}{*}{ Kunshan } & 1981 & 28.57 & 2.68 & 1.07 & 0.77 \\
\hline & 1991 & 26.55 & 4.56 & 1.11 & 0.80 \\
\hline & 2001 & 15.20 & 14.90 & 1.25 & 0.90 \\
\hline & 2011 & 16.25 & 13.43 & 1.29 & 0.93 \\
\hline & 2018 & 19.99 & 13.83 & 1.18 & 0.85 \\
\hline
\end{tabular}

The calculated values of CONTAG, SPLIT, SHDI and SHEI indicate that the number of small patches and degree of landscape fragmentation in all four study areas generally increased, with the landscape patches exhibiting an even distribution and the landscape patterns exhibiting increased fragmentation and uniformity during evolutions (Figure 7).

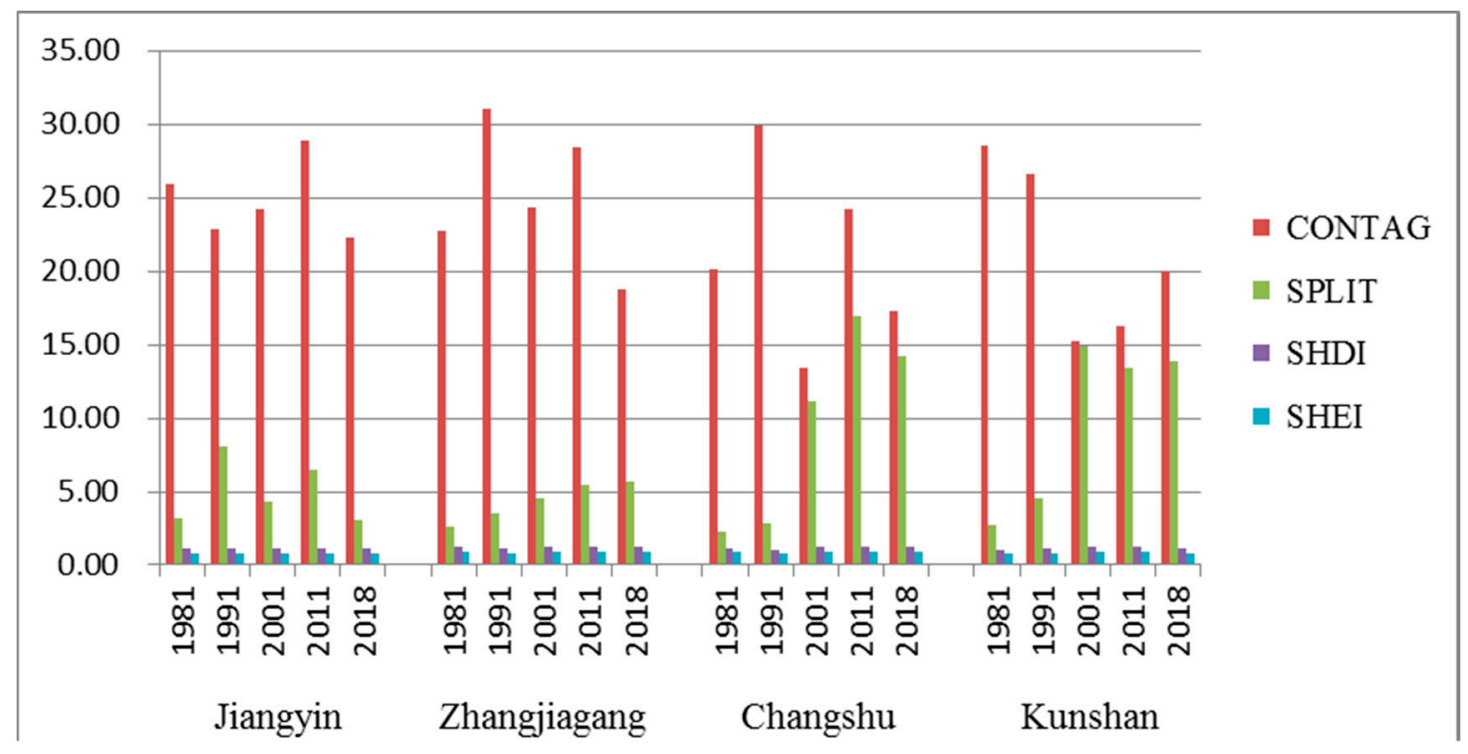

Figure 7. Calculation results from the landscape-level.

The CONTAG values of Jiangyin, Zhangjiagang and Changshu exhibited certain fluctuations, but remained relatively stable during 1981-2018, indicating that there was no dominant patch class that exhibited a high degree of contagion in these three study areas. The CONTAG values of Kunshan decreased continuously during 1981-2001 and increased continuously during 2001-2018, indicating a continuous decrease in the degree of contagion of the dominant patch class in Kunshan during 1981-2001, and a continuous increase during 2001-2018. 
The SPLIT values of Jiangyin fluctuated during 1981-2018, while those of Zhangiiagang, Changshu and Kunshan continuously increased. The SPLIT values of Changshu and Kunshan during 2001-2018 were approximately double those of Jiangyin and Zhangjiagang, indicating that the rates of landscape fragmentation in Zhangjiagang, Changshu and Kunshan were higher than that of Jiangyin, and the degree of landscape fragmentation in Changshu and Kunshan was higher than that in Jiangyin and Zhangjiagang.

The SHDI and SHEI values of the four study areas during 1981-2018 were relatively stable and exhibited a slight overall increase without significant changes, indicating an increase in the number of patch classes and landscape diversity. SHEI exceeded 0.77 in all four study areas during 1981-2018. SHEI ranges from 0 to 1 , with values closer to 1 indicating a higher degree of evenness in the distribution of the various landscape patch classes. Thus, the landscape patches of the four study areas were relatively evenly distributed. Cross-sectional comparisons were conducted and indicated that the SHDI and SHEI values of Kunshan exhibited the greatest rates of increase, indicating that Kunshan experienced the most significant changes in landscape diversity among the four study areas.

\section{Discussion}

During the analysis on landscape pattern indexes of industrial rural areas at the patch-class level and landscape-class level, this study discovered that:

(1) At the patch-class level, the landscape pattern evolution characteristics of industrial rural areas in Southern Jiangsu were that construction land had continuously encroached on green and cultivated land in all four study areas, exhibiting trends of expansion, centralization and the continuous consolidation of small patches into large patches during evolution and ultimately replacing cultivated land as the dominant landscape category. The result was in accordance with those in the studies of Yang, Sun et al., Ma et al., Xu et al. and Chuai et al. [31-35]. The reasons might be as follows: (1) Relevant studies showed that under the background of rapid socio-economic development, urban master planning is constantly updated, and relevant policies and regulations constantly promote human beings to expand the scope of urban built-up areas through deforestation, farmland reclamation and civil construction. Therefore, this study inferred that these human activities were likely to be the main reason for the changes of the area with urban landscape patches and the PLAND index [31]; and (2) in recent years, the process of urbanization development had been observed to show a strong aggregation (that is, all kinds of land shrink to the city center), and all kinds of land in the urban area were forced to "squeeze" out of the core area of the city. This phenomenon reflected in the landscape pattern in that the number of urban land patches had been reduced and the complexity increased [32].

(2) At the landscape-class level, the landscape patches were evenly distributed, and the landscape patterns exhibited increased fragmentation and uniformity during evolution. The evolution of landscape patterns in the four study areas was most intense during 1981-2001. Among the four study areas, Kunshan exhibited the most significant landscape pattern evolution characteristics. This result was also in accordance with the results of the studies by Yang, Sun et al., Ma et al., $\mathrm{Xu}$ et al. and Chuai et al. [31-35]. The reason for the results might be as follows: (1) The gradual improvement of a traffic network would cause disorderly cutting of the original patch landscape, which seriously affects the circulation of the ecological function of the regional landscape. From the perspective of landscape pattern, it is embodied in the increase of the SPILT index and the decrease of the CONTAG index [33,34]; and (2) for the rapid urbanization in China, the area of urban construction land had increased sharply, and the landscape tends to be homogeneous and fragmented. These might mainly explain the decline of the SHDI index and the SHEI index [35].

In order to help achieve sustainability for this region by improving the landscape pattern and promoting the development of ecological environment and tourism, the factors driving landscape 
pattern changes in the four study areas were analyzed to investigate the forces driving landscape pattern evolution in the industrial rural areas of Southern Jiangsu.

During the urbanization of the Southern Jiangsu region, triggered by China's economic reform, a series of economic support policies that were implemented by the central and local governments to meet the needs of economic development promoted the development of township enterprises and industries in Southern Jiangsu. This then boosted investments and economic prosperity, which attracted labor to the region and increased the demand for land utilization [36]. Consequently, the continuous aggregation of construction land patches led to the encroachment and segmentation of patches of other landscape categories, driving the evolution of landscape patterns in the industrial rural areas of Southern Jiangsu during 1981-2001. During the period of 1981-2001, a sort of significant event occurred, which pushed the industry and economic development hardly. Since the cold war in 1981, China improved the relationship with the West, especially the USA and Japan, the first and second developed countries in the world. China's economic reform began in 1978 and its market economy had begun to be gradually established since 1991. Pudong New District was set up in Shanghai in 1992. Hong Kong's return in 1997 has enhanced China's overall financial environment. Under this background, foreign capital and private capital flowed into Southern Jiangsu and accelerated the development of industry and enterprises in the study area. With the increase in the number and expansion of township enterprises in Southern Jiangsu, the urban and rural areas between industrial and agricultural populations became increasingly less distinct. This overcame the urban-rural economic divide, which drove the progress of urban-rural integration in Southern Jiangsu, resulting in the continuous aggregation and development of industrial rural areas and towns. However, such industrial rural development has been accompanied by the undesirable consequence of "smoke billowing from every village, factories emerging at every corner" [37]. To resolve this, the local governments in Jiangsu Province requested the establishment of industrial parks in townships, which led to further modifications to urban-rural structures in Southern Jiangsu. With the implementation of these development plans, the originally fragmented construction land patches aggregated further, thereby driving the decrease in the degree of fragmentation of construction land patches [38]. Since the onset of the 21st Century, "coordinated urban-rural development planning" has become China's national strategy, and there is a consensus that the urban-rural divide should be overcome among various circles of society. Consequently, the planning of residential, industrial and agricultural spaces in the industrial rural areas of Southern Jiangsu has changed. Plans for residential, industrial, agricultural, ecological water systems and transportation projects in small towns and villages have been matched with the development of urban areas, which integrated resource allocation, public service facilities and industrial layouts in both urban and rural areas. The influence of these strategies is most significant in Kunshan owing to its proximity to Shanghai, China's largest city. This is consistent with the comparative analysis results described above, which indicates that changes in landscape diversity were more significant in Kunshan than those in the other three study areas.

Despite the adverse effects of urbanization in the industrial rural areas of Southern Jiangsu, the industrial development that has occurred over the past four decades should not be wholly negated, and overcorrection measures to restore the rural appearances of these areas, such as the reconstruction of old villages or the reversion of industrial land to cultivated land, are strongly discouraged. The industrial rural areas of Southern Jiangsu should strive to achieve a balance between economic development and ecological sustainability, and develop a landscape construction pathway that is best suited to its circumstances.

Based on the discussions above, the landscape pattern evolution of industrial rural areas in Southern Jiangsu was dominated by the increase of construction land, change of policies and overall plan, resulting in fragmentation and homogenization. We propose the following strategies for improving the landscape pattern and promoting the development of the ecological environment and tourism, which are helpful to achieving sustainability for this region.

(1) Conserve existing cultivated land patches, appropriately expand grassland and forest land patches 
The planning of cultivated landscapes and surrounding spaces in the industrial rural areas of Southern Jiangsu should focus on conservation. Agricultural tourism can be developed and landscapes can be enhanced by developing modern circular agriculture and establishing ecological farmsteads $[39,40]$. Additionally, the construction of facilities such as rural greenways and parks, grassland, and forest land patches can be expanded or even organically merged, thereby increasing the degree of landscape aggregation of these patches and reducing the dominance of construction land patches.

(2) Respect the four decades of historical progress, reasonably modify industrial landscapes

The industrial development of the rural areas of Southern Jiangsu is a collective memory that is unforgettable for the local residents. Instead of demolishing factories that have been closed due to pollution problems, the land where they are located can be modified to create post-industrial landscapes that integrate with the natural and rural landscapes. Through such modifications, the history of the area can be respected and the unique characteristics of industrial rural areas can be fully manifested.

(3) Capitalize on the economic and positional advantage, optimize urban-rural landscape spaces

As the industrial rural areas of Southern Jiangsu are located in the Yangtze River Delta Economic Region, which exhibits the highest degree of urbanization in China, the urban-rural integration progress in these areas has been relatively rapid. Therefore, industrial rural areas possess economic and positional advantages, despite the severe rural landscape encroachment by urban landscapes. These favorable conditions of the industrial rural areas enable the construction of landscape connectors between urban and rural areas, such as urban-rural greenways and urban forest parks, to establish appropriate transition spaces between urban and rural landscapes. Consequently, urban and rural landscape spaces can be reasonably delineated through multi-plan integration at city, county, town and village levels, to alleviate the encroachment on rural landscapes by urban landscapes.

\section{Conclusions}

Four county-level cities in Southern Jiangsu with large numbers of industrial rural areas, i.e., Jiangyin, Zhangjiagang, Changshu and Kunshan, were selected as study areas for this work, which employed GIS and RS techniques. Remotely-sensed image data of the four study areas acquired during 1981, 1991, 2001, 2011 and 2018, as well as eight landscape pattern indexes at the patch-class and landscape levels, were selected to investigate landscape pattern evolution in the industrial rural areas of Southern Jiangsu from a provincial perspective, which involved analyzing the evolution characteristics and determining the factors driving evolution. The following conclusions were drawn:

(1) At the patch-class level, construction land had continuously encroached on green and cultivated land in all four study areas, exhibiting trends of expansion, centralization and the continuous consolidation of small patches into large patches during evolution and ultimately replacing cultivated land as the dominant landscape category. Concurrently, green and cultivated land patches, which are high-quality landscape resources, were continuously segmented into a large number of small patches, which led to fragmentation during the evolution of green and cultivated land. The effects of human disturbance on water bodies should receive attention, even though they are less severe than those experienced by green and cultivated land. Urban master planning was being constantly updated, and relevant policies and regulations were constantly promoting human beings to expand the scope of urban built-up areas through deforestation, farmland reclamation and civil construction. The processes of urbanization development had been observed to show strong aggregation. And all kinds of lands in the urban area were forced to "squeeze" out of the core area of the city.

(2) At the landscape level, the number of small patches and degree of landscape fragmentation generally increased in all four study areas. The landscape patches were evenly distributed 
and the landscape patterns exhibited increased fragmentation and uniformity during evolution. The evolution of landscape patterns in the four study areas was most intense during 1981-2001. Among the four study areas, Kunshan exhibited the most significant landscape pattern evolution characteristics. The gradual improvement of traffic network would cause disorderly cutting of the original patch landscape, which seriously affected the circulation of the ecological function of the regional landscape. For rapid urbanization in China, the areas of urban construction land had increased sharply, and the landscape tends to be homogeneous and fragmented.

(3) The direct cause of landscape pattern evolution in the industrial rural areas of Southern Jiangsu was the encroachment and segmentation of green and cultivated land patches by construction land patches, and the dominant factors driving the changes in construction land patches in the industrial rural areas of Southern Jiangsu were the effects of land and population aggregation exerted by the development of township enterprises and rural industries.

(4) This study concluded the landscape pattern and evolution dynamic of industrial rural areas, providing relevant fields with methods to investigate the evolution dynamic of urban-rural industry during urbanization and propose strategies for improving the landscape pattern and promoting the development of the ecological environment and tourism. It would also serve as a reference for other developing countries in Asia for sustainability of urban and rural development during industrialization, which is helpful to achieve sustainability for this region.

For the long period of 37 years and great dynamic changes of administrative division in this region, this study was limited in the scale of county-level cities. In the future we will try to focus more on the relationship between urban and rural areas with multivariate analysis based on GIS and RS techniques, which can provide more cases for the sustainability of similar regions in developing countries of Asia.

Author Contributions: Conceptualization, C.W.; methodology, C.W.; software, Y.Z.; validation, Y.Z.; formal analysis, Y.Z.; investigation, Y.Z.; resources, Y.Z.; data curation, Y.Z.; writing—original draft preparation, Y.Z.; C.W.; writing-review and editing, C.W.; T.S.; visualization, T.S.; supervision, T.S.; project administration, C.W.; funding acquisition, C.W.

Funding: This research was funded by the Humanity and Social Sciences Youth Foundation of the Ministry of Education of China, grant number 18YJCZH167.

Acknowledgments: Special thanks to all the team members for their helpful comments.

Conflicts of Interest: The authors declare no conflict of interest.

\section{References}

1. Rongtian, Z.; Huafu, J.; Xiaolin, Z. Rural Development Types and Rurality in the Yangtze River Delta. J. Nanjing Norm. Univ. 2014, 37, 132-136.

2. Zhu, J.; Zhan, Y.; Han, C. The Way to Urbanization in Southern Jiangsu: Change and Innovation of Hudai Town; China Social Sciences Press: Beijing, China, 2008.

3. Zhou, X. Study on the Change of Rural Landscape in Urbanization Process: A Case Study of Southern Jiangsu Province. Ph.D. Thesis, Nanjing Normal University, Nanjing, China, 2006.

4. Huo, G. Analysis on Planning and Design of Rural Landscape in Sunan Area. Master's Thesis, Suzhou University, Suzhou, China, 2012.

5. Zhao, Q.; Guo, X.; Li, G. The Transformation of the Rural Space in Southern Jiangsu under the Dominance of Development Zones: A Case study of Suzhou industrial park. Mod. Urban Res. 2014, 5, 9-14.

6. Cucari, N.; Wankowicz, E.; De Falco, S.E. Rural tourism and Albergo Diffuso: A case study for sustainable land-use planning. Land Use Policy 2019, 82, 105-119. [CrossRef]

7. Cattaneo, T.; Giorgi, E.; Ni, M. Landscape, Architecture and Environmental Regeneration: A Research by Design Approach for Inclusive Tourism in a Rural Village in China. Sustainability 2019, 11, 128. [CrossRef]

8. Balan, M.; Burghelea, C. Rural tourism and its implication in the development of the Fundata Village. Procedia Soc. Behav. Sci. 2015, 188, 276-281. [CrossRef] 
9. Dolejs, M.; Nadvornik, J.; Raska, P.; Riezner, J. Frozen Histories or Narratives of Change? Contextualizing Land-Use Dynamics for Conservation of Historical Rural Landscapes. Environ. Manag. 2019, 63, 352-365. [CrossRef] [PubMed]

10. Prestia, G.; Scavone, V. Enhancing the Endogenous Potential of Agricultural Landscapes: Strategies and Projects for a Inland Rural Region of Sicily. In Smart and Sustainable Planning for Cities and Regions, Sspcr 2017; Bisello, A., Vettorato, D., Laconte, P., Costa, S., Eds.; Springer: Cham, Switzerland, 2018; pp. 635-648.

11. Gonzalez Alvarez, D. Rethinking tourism narratives on the cultural landscapes of Asturias (Northern Spain) from the perspective of Landscape Archaeology: Do archaeologists have anything to say? Landsc. Res. 2019, 44, 117-133. [CrossRef]

12. Gonzalez Diaz, J.A.; Celaya, R.; Fernandez Garcia, F.; Osoro, K.; Rosa Garcia, R. Dynamics of rural landscapes in marginal areas of northern Spain: Past, present, and future. Land Degrad. Dev. 2019, 30, 141-150. [CrossRef]

13. Qi, W.; Tang, C. Study on the Influencing Factors of Carbon Emission in Industrial Villages: A Case Study of Fenghuang Village, Xiaoshan City, Hangzhou. Archit. Cult. 2018, 42, 174-178.

14. Li, H.; Wu, J.; Zhang, X.; Li, C. The Distribution Characteristics and Mechanism of the Rural Industrial Land with the "Southern Jiangsu Pattern"-A Case Study of Changshu. Econ. Geogr. 2018, 38, 152-159.

15. Liu, L.; Tang, X.; Xiong, X.; Du, J.; Wang, J. Ecological sensitivity analysis of rural natural landscape in the south of Jiangsu Province based on GIS. J. Nanjing For. Univ. 2018, 42, 159-164.

16. Zhao, Y.; Wu, X.; Huang, X. Research on the performance assessment of rural water landscape in South of Jiangsu. J. Nanjing For. Univ. 2018, 42, 174-178.

17. Dadashpoor, H.; Azizi, P.; Moghadasi, M. Land use change, urbanization, and change in landscape pattern in a metropolitan area. Sci. Total Environ. 2019, 655, 707-719. [CrossRef] [PubMed]

18. Guo, L.; Di, L.; Tian, Q. Detecting spatio-temporal changes of arable land and construction land in the Beijing-Tianjin corridor during 2000-2015. J. Geogr. Sci. 2019, 29, 702-718. [CrossRef]

19. Jiang, G.; Ma, W.; Zhou, D.; Zhao, Q.; Zhang, R. Agglomeration or dispersion? Industrial land-use pattern and its impacts in rural areas from China's township and village enterprises perspective. J. Clean. Prod. 2017, 159, 207-219. [CrossRef]

20. Van der Sluis, T.; Pedroli, B.; Frederiksen, P.; Kristensen, S.B.; Busck, A.G.; Pavlis, V.; Cosor, G.L. The impact of European landscape transitions on the provision of landscape services: An explorative study using six cases of rural land change. Landsc. Ecol. 2019, 34, 307-323. [CrossRef]

21. Stokes, E.C.; Seto, K.C. Characterizing and measuring urban landscapes for sustainability. Environ. Res. Lett. 2019, 14. [CrossRef]

22. Xi, Y.; Nguyen Xuan, T.; Li, C. Spatio-Temporal Variation Analysis of Landscape Pattern Response to Land Use Change from 1985 to 2015 in Xuzhou City, China. Sustainability 2018, 10, 4287. [CrossRef]

23. Wang, S.; Yang, K.; Yuan, D.; Yu, K.; Su, Y. Temporal-spatial changes about the landscape pattern of water system and their relationship with food and energy in a mega city in China. Ecol. Model. 2019, 401, 75-84. [CrossRef]

24. Ma, L.; Bo, J.; Li, X.; Fang, F.; Cheng, W. Identifying key landscape pattern indexes influencing the ecological security of inland river basin: The middle and lower reaches of Shule River Basin as an example. Sci. Total Environ. 2019, 674, 424-438. [CrossRef]

25. Li, Y.; Xue, C.; Shao, H.; Shi, G.; Jiang, N. Study of the Spatiotemporal Variation Characteristics of Forest Landscape Patterns in Shanghai from 2004 to 2014 Based on Multisource Remote Sensing Data. Sustainability 2018, 10, 4397. [CrossRef]

26. Jiangsu Statistical Bureau. Statistical Yearbook of Jiangsu 1991; China Statistical Press: Beijing, China, 1991.

27. Jiangsu Statistical Bureau. Statistical Yearbook of Jiangsu 2002; China Statistical Press: Beijing, China, 2002.

28. Jiangsu Statistical Bureau. Statistical Yearbook of Jiangsu 2012; China Statistical Press: Beijing, China, 2012.

29. Jiangsu Statistical Bureau. Statistical Yearbook of Jiangsu 2018; China Statistical Press: Beijing, China, 2018.

30. O'neill, R.V.; Krummel, J.R.; Gardner, R.E.; Sugihara, G.; Jackson, B.; DeAngelis, D.L.; Milne, B.T.; Turner, M.G.; Zygmunt, B.; Christensen, S.W.; et al. Indices of Landscape Pattern. Landsc. Ecol. 1988, 1, 153-162. [CrossRef]

31. Yang, W. Spatiotemporal change and driving forces of urban landscape in Beijing. Acta Ecol. Sin. 2015, 35, 4357-4366.

32. Sun, K.; Yang, Y.; Zhao, P.; Zhang, Z. Spatial Temporal Evolution of Landscape Pattern in Xi'an Based on $3 S$ Technology. J. Northwest For. Univ. 2015, 30, 180-185. 
33. Ma, S.; Zhang, Y.; Sun, C. Optimization and Application of Integrated Land Use and Transportation Model in Small- and Medium-Sized Cities in China. Sustainability 2019, 11, 2555. [CrossRef]

34. Xu, L.; Chen, S.S.; Xu, Y.; Li, G.; Su, W. Impacts of Land-Use Change on Habitat Quality during 1985-2015 in the Taihu Lake Basin. Sustainability 2019, 11, 3513. [CrossRef]

35. Chuai, X.; Wen, J.; Zhuang, D.; Guo, X.; Yuan, Y.; Lu, Y.; Zhang, M.; Li, J. Intersection of Physical and Anthropogenic Effects on Land-Use/Land-Cover Changes in Coastal China of Jiangsu Province. Sustainability 2019, 11, 2370. [CrossRef]

36. Hu, M. Development Process and Enlightenment of Township Enterprises in China since Reform and Opening: The Case of Township Enterprises in Jiangsu from 1978 to 1992. Lit. Chin. Communist Party 2008, 4, 24-29.

37. Yu, G.; Li, Y. Present Main Problems of Township Enterprises: Investigation and Research of Township Enterprises in Jiangsu Province from 1978-1992. Issues Agric. Econ. 1989, 10, 24-29.

38. Sun, X. Research on the Development Model of Urbanization of Village Area in Suzhou. Master's Thesis, Suzhou University of Science and Technology, Suzhou, China, 2017.

39. Zhong, Z.; Chen, Z.; Xu, Y.; Ren, C.; Yang, G.; Han, X.; Ren, G.; Feng, Y. Relationship between Soil Organic Carbon Stocks and Clay Content under Different Climatic Conditions in Central China. Forests 2018, 9, 598. [CrossRef]

40. Dax, T.; Zhang, D.; Chen, Y. Agritourism Initiatives in the Context of Continuous Out-Migration: Comparative Perspectives for the Alps and Chinese Mountain Regions. Sustainability 2019, 11, 4418. [CrossRef]

(C) 2019 by the authors. Licensee MDPI, Basel, Switzerland. This article is an open access article distributed under the terms and conditions of the Creative Commons Attribution (CC BY) license (http://creativecommons.org/licenses/by/4.0/). 